\title{
Engineered Particulates for Co-Firing of Diverse Feedstocks
}

Final Report

Start Date: September 30, 2002

End Date: September 29, 2003

Joseph J. McCarthy, Kunal Jain, Hongming Li, and Deliang Shi March 2004

DE-FG26-02NT41554

University of Pittsburgh

1249 Benedum Hall Pittsburgh, Pennsylvania 15261

Ph: 412-624-7362, Fax: 412-624-9639, email: jjmcc@ pitt.edu 


\section{DISCLAIMER}

"This report was prepared as an account of work sponsored by an agency of the United States Government. Neither the United States Government nor any agency thereof, nor any of their employees, makes any warranty, express or implied, or assumes any legal liability or responsibility for the accuracy, completeness, or usefulness of any information, apparatus, product, or process disclosed, or represents that its use would not infringe privately owned rights. Reference herein to any specific commercial product, process, or service by trade name, trademark, manufacturer, or otherwise does not necessarily constitute or imply its endorsement, recommendation, or favoring by the United States Government or any agency thereof. The views and opinions of authors expressed herein do not necessarily state or reflect those of the United States Government or any agency thereof." 


\section{ABSTRACT}

The goal of this project is to develop a novel methodology for the formation of engineered particulates of energy-relevant material. Specifically, we aim to control interparticle cohesion in such a way as to generate macro-particles or agglomerates of several differing types of primary particles in specific proportions such that they would be of utility for co-firing applications. In Phase I of this project, we used a combination of experimentation and simulation to validate theoretically derived mixing/segregation rules for cohesive granular materials in static systems, flowing systems, and gas-solid systems. 


\section{TABLE OF CONTENTS}

Contents

EXECUTIVE SUMMARY 1

$\begin{array}{ll}\text { INTRODUCTION } & 3\end{array}$

EXPERIMENTAL

Discrete Modeling . . . . . . . . . . . . . . . . . . . . 3

Capillary Forces . . . . . . . . . . . . . . . . . . . . . . 4

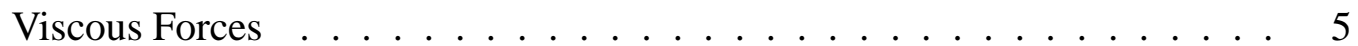

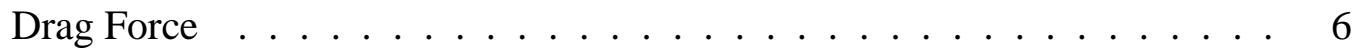

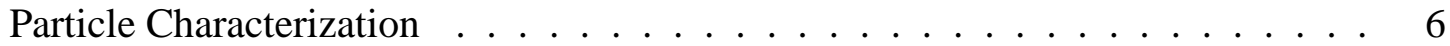

RESULTS AND DISCUSSION 9

The Granular Capillary Number . . . . . . . . . . . . . . . . . . . . . 9

Mixing/Segregation in Gas-solid and Shearing Flows . . . . . . . . . . . 10

CONCLUSIONS

REFERENCES 


\section{EXECUTIVE SUMMARY}

The goal of this project is to develop a novel methodology for the formation of engineered particulates of energy-relevant material. Specifically, we aim to control interparticle cohesion in such a way as to generate macro-particles or agglomerates of several differing types of primary particles in specific proportions such that they would be of utility for co-firing applications. Specific goals of the Phase I project were as follows:

1. Develop a characterization tool for gas-solid flows.

2. Extend our agglomeration theory to include shearing effects $(\mathrm{Co})$ and fluid drag (characterization tool for gas-solid flows from goal 1) for increased control of "phase" behavior.

3. Run computational/experimental agglomeration tests to validate our tools/theory.

In the course of the project we worked extensively on cohesive characterization tools. We extended the Collision Number ( $\mathrm{Co}$ - the ratio of cohesive to shearing forces) to be useful for non-monodisperse systems, and developed both the mono- and polydisperse Granular Capillary Number $\left(\mathrm{Ca}_{g}\right)$ for gas-solid systems. In order to develop a characterization tool for gas-solid flows we examined the ratio of the drag force of the particles (Equation 13) to the maximum capillary force (given as $F_{c_{\max }}=2 \pi R \gamma$ ). This yields the Granular Capillary Number $(\mathrm{Ca})$ as

$$
C a_{g}=\frac{F_{c}}{F_{d}}=\frac{4 \gamma}{C_{d} \rho_{g} R\left|u-v_{p}\right|\left(u-v_{p}\right) \varepsilon^{-\chi+1}} .
$$

In order to modify both the $C o$ and $C a_{g}$ to be applicable to binary systems we need to treat one as a "guest" particle, and proceed as follows. In both sheared beds and gassolid flows, the proper capillary force is based on the geometric mean radius. For gas-solid flows, the determination of which of the particles in the pair is to be thought of as the guest is obtained by establishing the one that is most effected by the fluid drag force (i.e., whichever one maximizes $F_{d_{i}} / W_{i}$ ). In the case of sheared granular beds the calculation is slightly more complex. One can envision that, even in the case of two similar particles being stuck together, that the third particle - the one creating the collision force via collision with the binary pair - may be of either particle type. This means that in the case of calculating the collision number between two similar particles, $\mathrm{Co}_{i i}$, the collision force $\left(F_{c o l}\right)$ used in the denominator is the larger of $F_{c o l}$ and $F_{c o l}$. Once again, as in the gassolid flows, the choice of guest particle for the calculation of the collision number between dissimilar particles, $C o_{i j}$, is then determined by which of them maximizes $F_{c o l} / W_{i}$, where $F_{c o l}$ is determined as with the $C o_{i i}$ calculation.

Experiments were performed in static and sheared beds, while simulations were performed for gas-solid systems. In all cases, results were in agreement with predictions 
from characterization tool-based theories, showing a level of control of mixing/segregation which was previously unattainable. This work has set the stage for working with energyrelevant materials in mixing/segregation experiments under shearing and fluidization/conveying conditions. 


\section{INTRODUCTION}

Recently, for reasons of both energy efficiency and environmental expediency, there has been an increased interest in combining differing fuels - such as coal, char, biomass, limestone, etc. - in combustion-power based systems. In these systems, it is imperative that flows are both continuous and uniform for proper operation of the combustors; however, most, if not all, of the feedstocks which have been proposed as potential coal co-firing agents have complex particle shapes and very different sizes and properties when compared to conventional fuels. Moreover, many of these materials tend to be cohesive due to high moisture content and/or small particle size. These factors contribute to making the attainment of consistent concentration and flow in co-firing plants technologically challenging and often lead to inefficient and inconsistent operation of combustion systems.

A growing trend in particle technology is the development of engineered particulates or controlled agglomerates [1,2]. Engineered particulates are ideally suited for co-firing applications, being macro-particles or agglomerates of several differing types of primary particles in specific proportions. Typical methodologies for producing engineered particles, however, are unsuitable for co-firing application (either because of expense or due to limitations on material characteristics). The ultimate goal of the current project was to develop a methodology for the formation of engineered particulates via a novel technique such that we may form energy-relevant controlled agglomerates for use in the co-firing of diverse feedstocks. It is expected that developing these engineered particulates will result in easier handling and more consistent and reliable transport and flow of diverse feedstocks, thus allowing greater efficiency for co-fired combustion systems.

A combination of discrete simulation and experimentation were used toward attaining this goal. First, we developed a powerful Discrete Element Method computational code that is capable of incorporating cohesive particle interactions into static, sheared, and gas-solid flows. Moreover, we devised simple, discrete-level characterization tools for cohesive granular solids in dense gravity-driven flows or sheared flows as well as gas-solid flows (i.e., cohesion vs. particle weight $\left(\mathrm{Bo}_{g}\right)$; cohesion vs. shear $(\mathrm{Co})$; and cohesion vs. fluid drag $\left.\left(C a_{g}\right)\right)$. We, then, exploited these characterization tools to build a theoretical approach that enables us to predict the behavior of mixtures of differing particle types. This approach has been tested both experimentally and using DEM simulations for several differing types of flow.

\section{EXPERIMENTAL}

\section{Discrete Modeling}

A powerful modeling technique for flow of particulate materials is that of Particle Dynamics Simulation (PDS) also known as the Discrete Element Method (DEM). In this technique the bulk flow of the material is captured via simultaneous integration of the interaction 
forces between individual pairs of particles [3]. Our group has a strong expertise in the use of these types of models. In particular, we have used these techniques to perform computational experiments on model systems which has allowed us to complement and even improve physical experiments as well as other modes of simulation. Moreover, we have extended our models to incorporate cohesive particle interactions.

Particle Dynamics, a discrete method of simulation, has emerged as one of the most important tools in probing granular flows [4-10]. The method is extremely general in that Newton's second law of motion is used to determine the trajectories of individual particles and the time evolution of these trajectories then determines the global flow of the granular material. The equations that describe the particle motion, therefore, are:

\section{Linear Motion:}

$$
m_{p} \frac{d v_{p}}{d t}=-m_{p} g+F_{n}+F_{t}
$$

Angular Motion:

$$
I_{p} \frac{d \omega_{p}}{d t}=F_{t} R
$$

where $F_{n}$ and $F_{t}$ are the interparticle forces - normal and tangential, respectively - acting on the particle and are functions of contact, drag, pressure and cohesive interactions. The interparticle forces for cohesionless systems are typically determined from contact mechanics considerations, so that in their simplest form they include normal (often, Hertzian) [11] repulsion and some approximation of tangential friction [12]. In the present work, normal interactions are modeled as elasto-plastic contacts after the work of Thornton [13], while a single-parameter history-dependent friction is used in the tangential direction [14] - details can be found in Ref. [5].

\section{Capillary Forces}

Moisture is a common cause of cohesion in particle flows. Several models based on the solution of the Young-Laplace equation are available in the literature $[7,8,15]$ for the case when the degree of saturation is low enough that discrete bridges are present at the points of solid contact (pendular regime). The capillary force, $F_{c}$, due to both the surface tension of the bridge fluid as well as the pressure difference arising from neck curvature may be expressed as

$$
F_{c}=2 \pi r_{2} \gamma \sin \beta \sin (\beta+\theta)+\pi R^{2} \Delta P \sin ^{2} \beta
$$

where $r_{2}$ is the bridge neck radius, $\beta$ is the half filling angle, $\theta$ is the contact angle, $\gamma$ is the fluid's surface tension and $\Delta P$ is the pressure difference across the air-liquid interface. 
The pressure reduction across the capillary bridge is given by the Laplace equation:

$$
\Delta P=\gamma\left[\frac{1}{r_{1}}-\frac{1}{r_{2}}\right]
$$

where $r_{1}$ is the bridge meridional radius of curvature. Mikami et al. [8] provide an empirical fit to the numerical solution of the Laplace-Young equations expressed as

$$
\hat{F}=\exp (A \hat{h}+B)+C
$$

where

$$
\begin{gathered}
A=-1.1 \hat{V}^{-0.53} \\
B=(-0.34 \ln \hat{V}-0.96) \theta^{2}-0.019 \ln \hat{V}+0.48 \\
C=0.0042 \ln \hat{V}+0.0078
\end{gathered}
$$

where $\hat{F}$ is the normalized capillary force $\left(F_{c} / 2 \pi R \gamma\right) ; \hat{V}$ is the bridge volume made dimensionless by the particle radius $(R) ; 2 \hat{h}$ is the separation distance between the particle made dimensionless by the particle radius $(R)$; and $A, B$ and $C$ are empirical functions. In our simulations, the moisture content is assumed to be sufficiently low that bridges only form upon contact of the solid surfaces. These bridges remain in place, however, after solid contact has ceased, until the particles reach a critical separation (rupture) distance $\left(h_{c}\right)$ given by:

$$
\hat{h}_{c}=(0.62 \theta+0.99) \hat{V}^{0.34} .
$$

In order to avoid system-size effects as much as possible, no bridges are formed between the particles and any confining walls.

\section{Viscous Forces}

Dynamic formation/breakage of liquid bridges results in a viscous force resisting motion, derived from lubrication theory. It is essential that any liquid-induced cohesion simulation include these effects as they may become large relative to the capillary force as the particle velocity increases [16]. In the limit of rigid spheres, Adams and Perchard [17] derive the viscous force in the normal direction $\left(F_{v_{n}}\right)$ to be

$$
F_{v_{n}}=6 \pi \mu R v_{n} \frac{R}{2 h}
$$

where $\mu$ is the bridge fluid's viscosity, and $v_{n}$ is the relative normal velocity of the spheres. In the tangential direction $\left(F_{v_{t}}\right)$, Lian et al. [7] suggest the use of the the solution due to Goldman et al. [18] for the viscous force between a sphere and a planar surface 


$$
F_{v_{t}}=\left(\frac{8}{15} \ln \frac{R}{2 h}+0.9588\right) 6 \pi \mu R v_{t}
$$

where $v_{t}$ is the relative tangential velocity of the spheres.

\section{Drag Force}

Drag between the fluidizing medium (gas) and the particle(s) couples the discrete simulation to the (continuum) fluid flow and represents the primary mode of inter-phase momentum transfer. The drag force not only depends on the local fluid flow field but also on the presence of the neighboring particles [19].

In this work, we use the drag force $\left(F_{d}\right)$ suggested by Di Felice [20] in 1994. In the formulation

$$
F_{d}=\frac{1}{2} C_{d} \rho_{g} \pi R^{2}\left|u-v_{p}\right|\left(u-v_{p}\right) \varepsilon^{-\chi+1}
$$

where $u$ is the local gas velocity, $v_{p}$ is the particle velocity, $C_{d}$ and $\chi$ are functions of the particle Reynolds Number $\left(R e=\frac{2 R \rho_{g} \varepsilon\left(u-v_{p}\right)}{\mu_{g}}\right)$ and are given by

$$
\begin{gathered}
C_{d}=\left[0.63+\frac{4.8}{R e^{0.5}}\right]^{2} \\
\chi=3.7-0.65 \exp \left[-\frac{\left(1.5-\log _{10} R e\right)^{2}}{2}\right]
\end{gathered}
$$

\section{Particle Characterization}

Particulate materials tend to segregate (or unmix) when essentially any difference in mechanical properties of the particles is present (though size and density differences tend to be thought of as dominate driving forces for segregation). This fact can lead to significant difficulties in heterogeneous reburning by causing inconsistent feed amount and quality. Our group has a history in the mixing and segregation of particles. By leveraging this knowledge in the area of cohesive materials, we have been able to build a theoretical framework for understanding the mixing and segregation behavior of diverse, cohesive feedstocks where previously none has existed.

In previous work, we have developed discrete characterization tools for wet cohesive granular materials. The basis of our characterization models is the competition between various forces acting on individual particles within a granular bed (i.e., cohesion vs. particle weight $\left(B o_{g}\right)$ and cohesion vs. shear $\left.(\mathrm{Co})\right)$. The Granular Bond Number [5] $\left(B o_{g}\right)$, represents the ratio of the maximum capillary force and the weight of a particle. This group is dominant in characterizing the effects of cohesion in static or near-static systems. The ratio of maximum cohesive force and the collisional force due to Bagnold [21] is called 

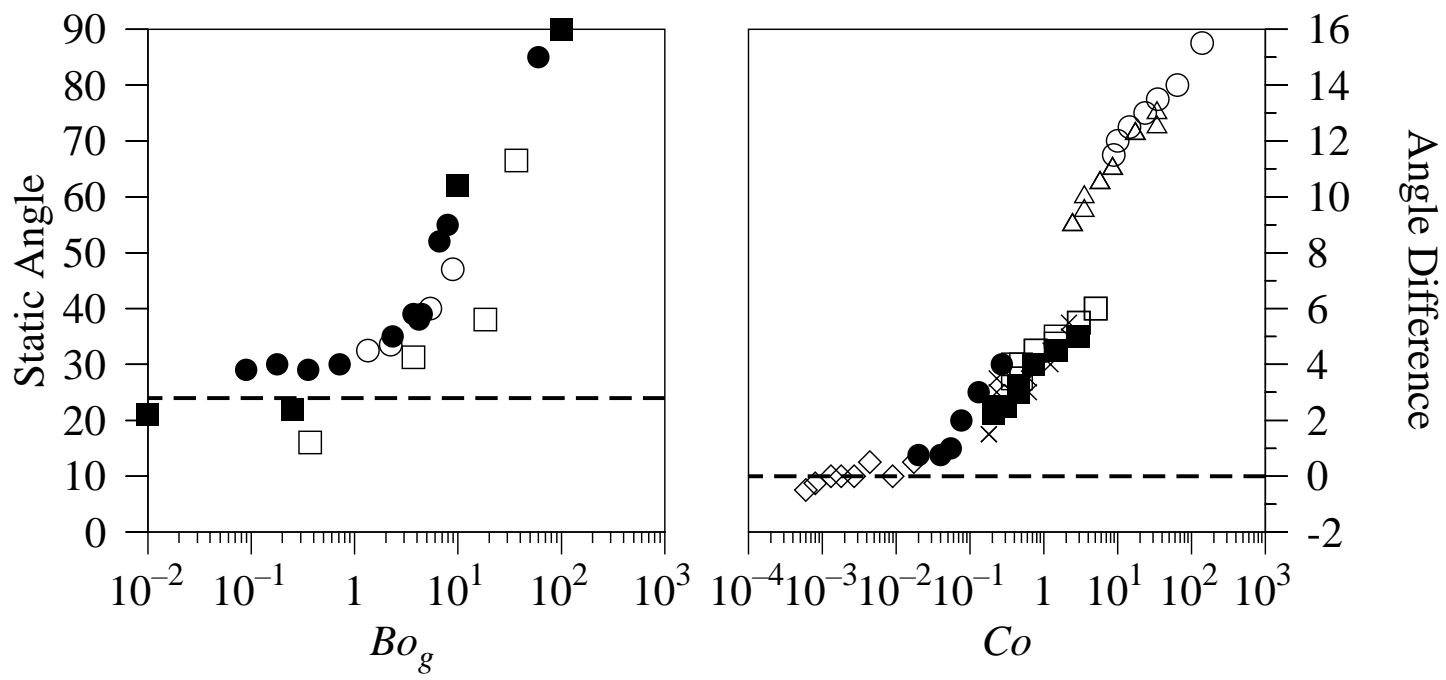

Figure 1: Monodisperse Characterization Tools for Static and Sheared Systems. Bo $g$ and $C o$ are shown to quantify the signifi cant differences in behavior of granular materials undergoing a freefbwing to cohesive materials transition.

the Collision Number [5] ( $\mathrm{Co})$. This number becomes dominant in highly sheared granular materials where $B o_{g}>1$.

As seen in Figure 1, both $\mathrm{Bo}_{g}$ (using heaping angle as a metric) and $\mathrm{Co}$ (using the change in dynamic angle in a tumbler as a metric) exhibit a clear distinction between a freeflowing behavior, evidenced by the asymptotic "flat" section (both curves must asymptote to the dashed line at zero cohesion), to cohesive behavior, evidenced by the angled sections. Note that these trends hold true for a variety of materials (differing symbols).

Also in a previous project, we extended the definition of the $B o_{g}$ to make it applicable for binary systems of particles. The modified Bond number determines the importance of cohesion relative to particle weight by considering one particle within a two particle grouping as a "guest" particle (obviously, the less massive of the two) so that it is defined as

$$
B o_{g}=\frac{F_{c}}{W_{\text {guest }}}=\frac{2 \pi \gamma R_{e f f}[\cos (\theta)]_{\min }}{\frac{4}{3} \pi g\left(R^{3} \rho_{s}\right)_{\min }}=\frac{3 \gamma R_{e f f}[\cos (\theta)]_{\min }}{2 g\left(R^{3} \rho_{s}\right)_{\min }},
$$

where $F_{c}$ is the cohesive force, $\gamma$ is the interstitial fluid's surface tension, the particle weight $(W)$ to be used is that of the less massive particle, $\rho_{s}$ is the particle density (again, of the less massive particle), $g$ is the acceleration of gravity, $\theta$ is the wetting angle, and differing wetting characteristics are incorporated simply by using the larger value of $\theta$ (or the smaller value of $\cos (\theta)$ ). Note also that while $R$ is the radius of the less massive particle, $R_{\text {eff }}$ is the effective particle radius given as the geometric mean of the particle radii, 


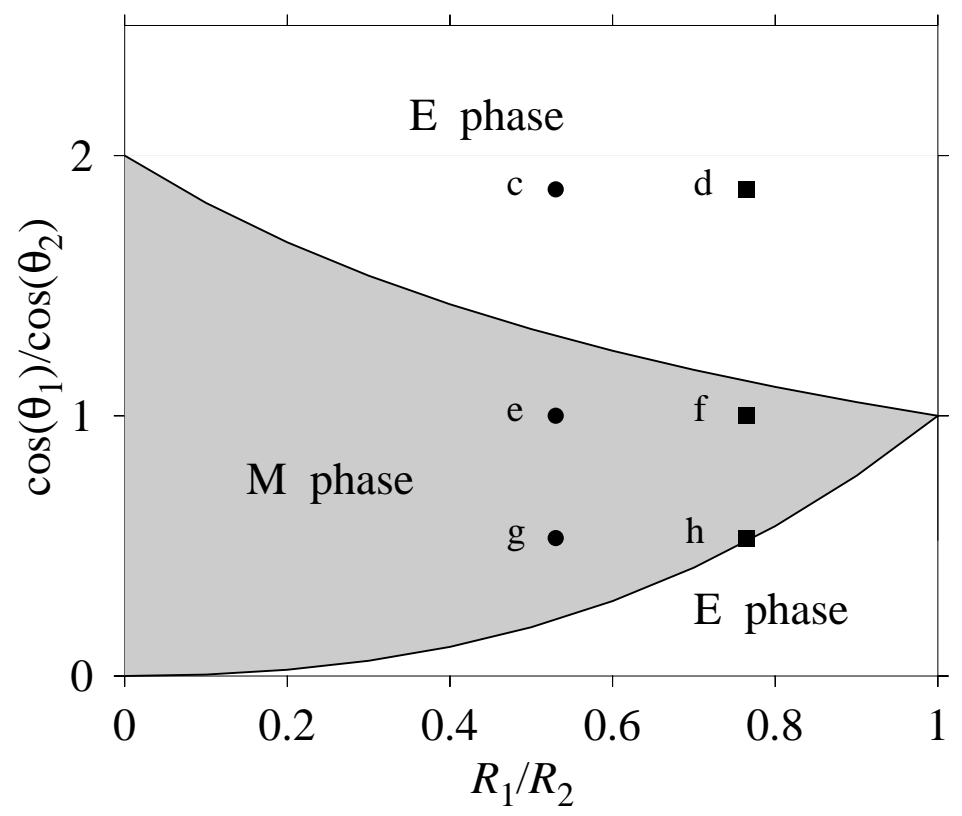

Figure 2: Phase Diagram for Cohesive Mixing/Segregation in "Static" Systems. Diagram outlining cohesive mixing/segregation behavior for a binary system with identical densities, but different sizes and surface properties. Regions where interstitial moisture mitigates segregation are labeled as the $\mathrm{M}$ phase (dark) and those where it enhances segregation are the E phase (light).

$R_{e f f}=2 R_{1} R_{2} /\left(R_{1}+R_{2}\right)$.

This definition allowed us to develop a theory (see Figure 2) that allows us to outline for the first time - when cohesive (wet) systems will mix or segregate relative to the freeflowing (dry) case based on the particles' mechanical and surface properties. Specifically, in a $B o_{g}$ dominated flow (i.e., low shear and no significant fluid drag), we can determine the steady-state behavior of the system by comparing the value of the $B o_{g}$ for each potential particle grouping (1-1 or 1-2 or 2-2) within the system in order to determine the "thermodynamically" favored of the three groupings (i.e., which of the three $B o_{g}$ numbers is largest). In this way we can determine under what conditions we can form 1-1, 2-2, or 1-2 dominated agglomerates (see Figure 2). In the case where the 1-2 interaction is dominant we predicted that the materials would mix to a greater extent when made cohesive (M-phase in the figure); otherwise, cohesion would have a negative impact (E-phase in the figure). Experiments (Figure 3) done in our lab support this theory, where particle systems that would otherwise mix can be made to segregate, and vice versa simply by adding moisture [22]. This theory was the basis of the Phase I project and has motivated the continuation as a Phase II candidate. These existing tools have been tested for prototypical wet particulate matter (glass beads with water) and are easily applicable to a wide variety of feedstocks simply by changing the individual particle properties (density, stiffness, particle size, etc.). 

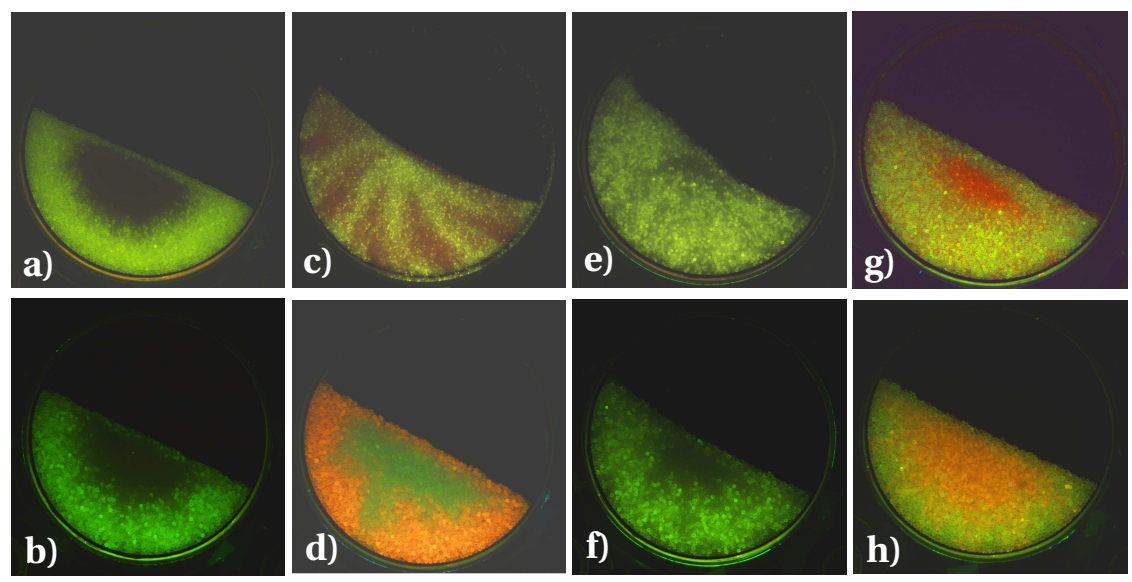

Figure 3: Controlled Mixing and Segregation in "Static" Systems. The top and bottom rows correspond to size ratios of 0.53 and 0.75 , respectively. From left to right the images represent results when dry - (a) and (b); wet with the larger particle (red/orange) being more hydrophobic - (c) and (d); wet with both particles hydrophilic - (e) and (f); and wet with the smaller particle (red/orange) being more hydrophobic - $(\mathrm{g})$ and $(\mathrm{h})$. Note that when the smaller particle (red/orange) is more hydrophobic - (g) and (h) - the two size ratios respond differently, as predicted by our theory.

\section{RESULTS AND DISCUSSION}

\section{The Granular Capillary Number}

In order to develop a characterization tool for gas-solid flows we examined the ratio of the drag force of the particles (Equation 13) to the maximum capillary force (given as $\left.F_{c_{\max }}=2 \pi R \gamma\right)$. This yields the Granular Capillary Number $\left(\mathrm{Ca}_{g}\right)$ as

$$
C a_{g}=\frac{F_{c}}{F_{d}}=\frac{4 \gamma}{C_{d} \rho_{g} R\left|u-v_{p}\right|\left(u-v_{p}\right) \varepsilon^{-\chi+1}} .
$$

In order to test the utility of this characterization tool, we examined both the onset of fluidization and the mixing within monodisperse fluidized beds. The minimum fluidization velocity is typically defined as the velocity at which the bed pressure drop goes through a maximum value. A critical component of this definition is that, while the pressure drop is ultimately determined solely by the weight of the fluidized particles, the value of the pressure drop can exceed this limit prior to fluidization. In the small fluidization systems examined here a simpler, but equivalent, definition of the minimum fluidization velocity is used. The approach used for determining the minimum fluidization velocity is similar in spirit to that followed by Kafui et al. [23] which is based on monitoring the state of the particle connectivity network. We find that, using this definition of the minimum fluidization velocity, an increase in the $\mathrm{Ca}_{g}$ (surface tension) increases the velocity necessary to achieve 


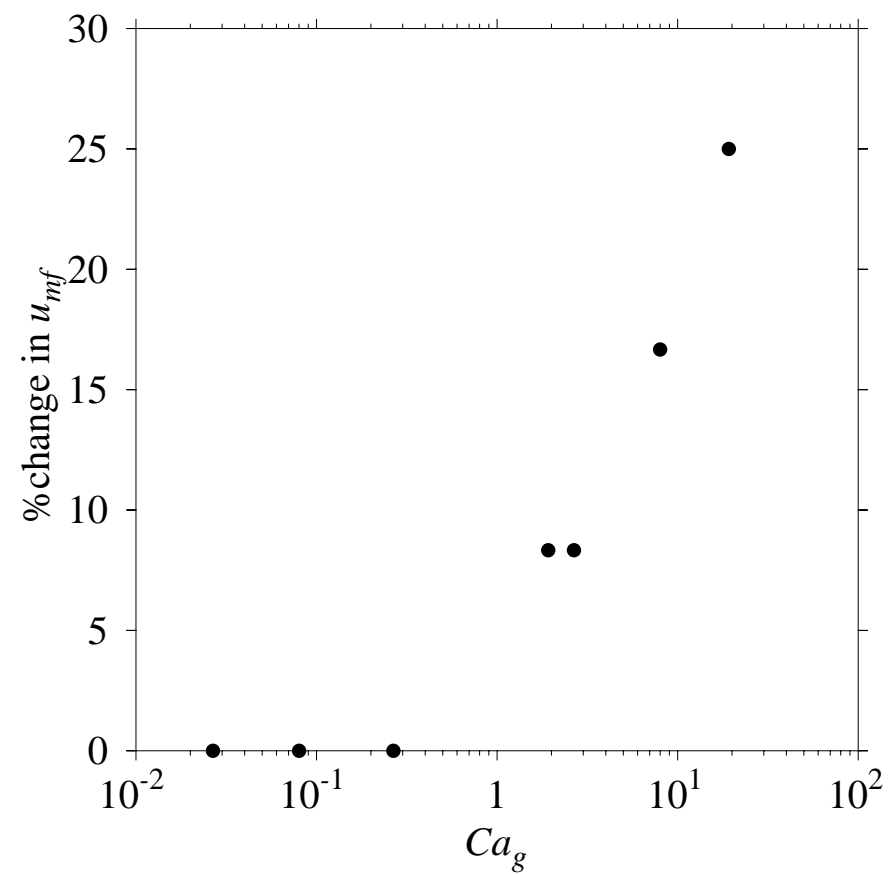

Figure 4: Increased Fluidization Velocity Versus Granular Capillary Number. Plotting the values of the minimum fluidization velocity versus $\mathrm{Ca}$ g shows a clear transition point where this value increases dramatically.

a fluidized system relative to that of the completely dry (non-cohesive) case. Figure 4 shows a plot of the percentage increase in the minimum fluidization velocity as a function of the $C a_{g}$. For values of surface tension where the $C a_{g}<1$, changes in the fluidization velocity from that of a completely dry granular material are essentially unmeasurable; however, for larger surface tensions, where the values of $C a_{g}>1$, the fluidization velocities increases markedly requiring as much as a $30 \%$ increase in $u_{m f}$ at the highest $C a_{g}$ examined.

We next examined changes of the mixing rate of mechanically identical particles with changes in gas velocity as well as liquid bridge surface tension. Plotting the resultant mixing rate constants as a function of $C a_{g}$, in Figure 5, shows that this assertion is valid. That is, mixing rates are high for small $C a_{g}$ and drop dramatically as $C a_{g}$ increases.

\section{Mixing/Segregation in Gas-solid and Shearing Flows}

In order to utilize similar arguments to those used to develop mixing/segregation phase diagrams in Section, we first needed to modify the $C o$ and $C a_{g}$ to be applicable to binary systems. Keeping with the idea that the proper usage for dissimilar particles is to treat one as a "guest" particle, we proceed as follows. In both sheared beds and gas-solid flows, the proper capillary force is still that used in Section, i.e., one that uses the geometric mean radius. For gas-solid flows, the determination of which of the particles in the pair is to be 


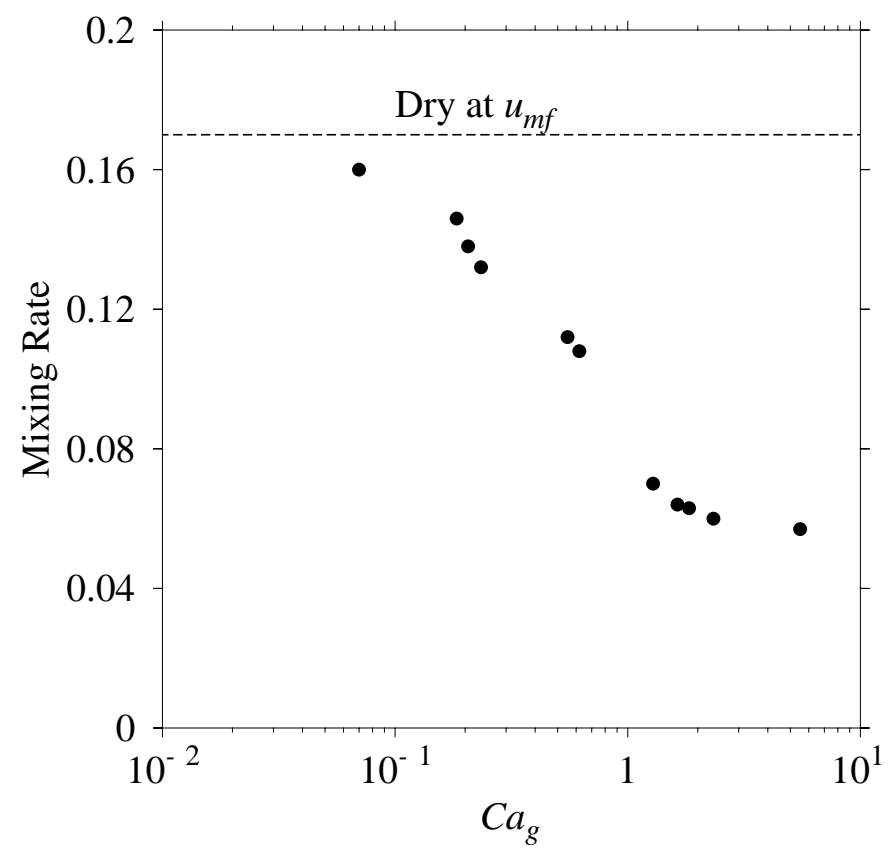

Figure 5: Mixing Rate Versus Granular Capillary Number. Plotting the mixing rate as a function of $\mathrm{Ca}$ causes the data from trials that vary both the fluidization velocity as well as the surface tension to collapse on one curve.

thought of as the guest is obtained by establishing the one that is most effected by the fluid drag force (i.e., whichever one maximizes $F_{d_{i}} / W_{i}$ ).

In the case of sheared granular beds the calculation is slightly more complex. One can envision that, even in the case of two similar particles being stuck together, that the third particle - the one creating the collision force via collision with the binary pair - may be of either particle type. This means that in the case of calculating the collision number between two similar particles, $\mathrm{Co}_{i i}$, the collision force $\left(F_{c o l}\right)$ used in the denominator is the larger of $F_{c o l}$ and $F_{c o l}$. Once again, as in the gas-solid flows, the choice of guest particle for the calculation of the collision number between dissimilar particles, $C o_{i j}$, is then determined by which of them maximizes $F_{c o l} / W_{i}$, where $F_{c o l}$ is determined as with the $C o_{i i}$ calculation.

Now that binary characterization tools for wet gas-solid and sheared flows have been established for both gas-solid and shear flows, one can simply analytically compare the three resulting numbers (e.g., $\mathrm{Co}_{11}$ vs. $\mathrm{Co}_{22}$ vs. $\mathrm{Co}_{12}$ ) to determine what conditions lead to cohesion aiding mixing (where $\mathrm{Co}_{12}$ is largest) and which hamper mixing (when either $\mathrm{Co}_{11}$ or $\mathrm{Co}_{22}$ is largest). In doing so, we can then develop phase diagram to describe this behavior as shown in Figures 6 and 7.

Although extending the degree of validation of these tools - both for prototypical par- 

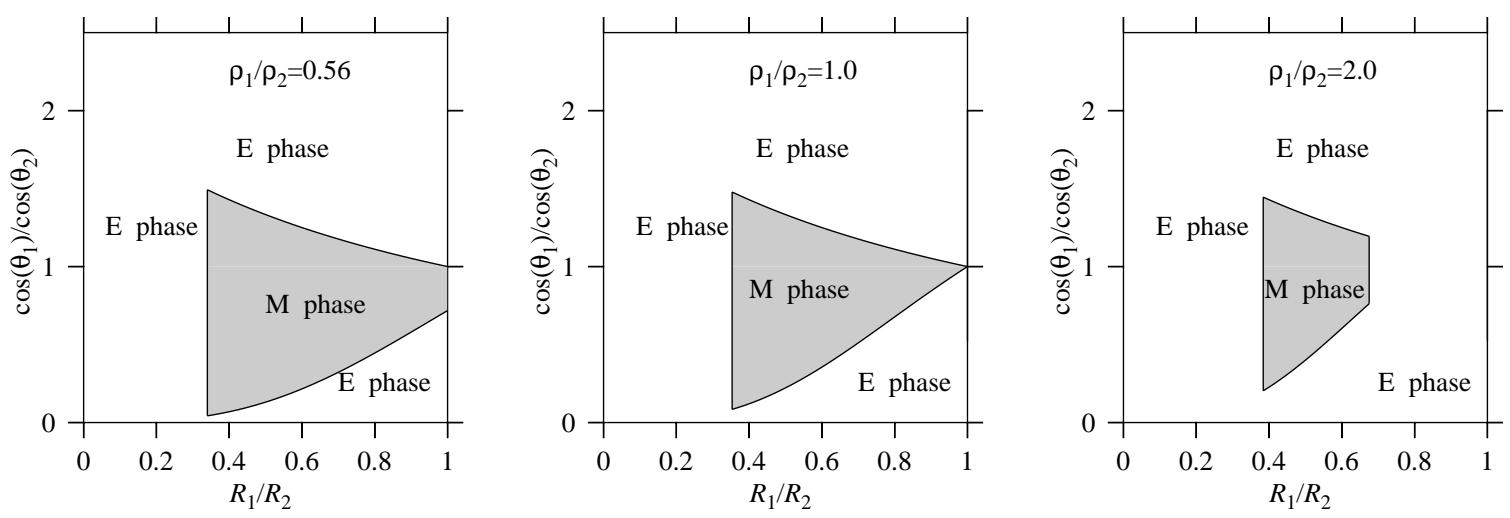

Figure 6: Phase Diagram for Cohesive Mixing/Segregation in Sheared Systems and Several Density Ratios. Again, regions where interstitial moisture mitigates segregation are labeled as the $\mathrm{M}$ phase (dark) and those where it enhances segregation are the E phase (light). Note that in all cases the $\mathrm{M}$ phase is smaller than for "static" systems.

ticulate materials (like glass beads) and energy-relevant materials - is the subject of future work, there has been a limited degree of validation to date. Experiments in an annular shear cell - run to steady state - yield results in agreement with predicted trends for sheared beds of materials (see Figure 8). Similarly, simulations of binary gas-solid flows agree reasonably well with our theory (see Figure 9).

\section{CONCLUSIONS}

In the course of the project we worked extensively on cohesive characterization tools. We extended the Collision Number ( $\mathrm{Co}$ ) to be useful for non-monodisperse systems, and developed both the mono- and polydisperse Granular Capillary Number $\left(\mathrm{Ca}_{g}\right)$ for gassolid systems. Experiments were performed in static and sheared beds, while simulations were performed for gas-solid systems. In all cases, results were in agreement with predictions from characterization tool-based theories, showing a level of control of mixing/segregation which was previously unattainable. This work has set the stage for working with energy-relevant materials in mixing/segregation experiments under shearing and fluidization/conveying conditions. 

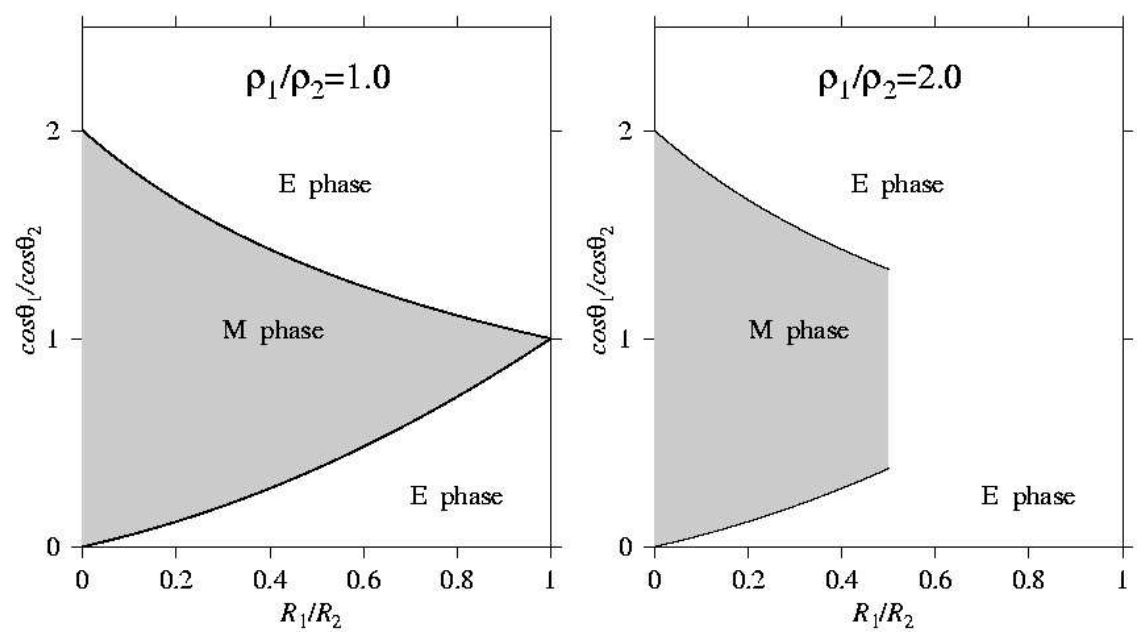

Figure 7: Phase Diagram for Cohesive Mixing/Segregation in Sheared Systems and Several Density Ratios. As with the sheared systems, the M phase is slightly smaller than for "static" systems.
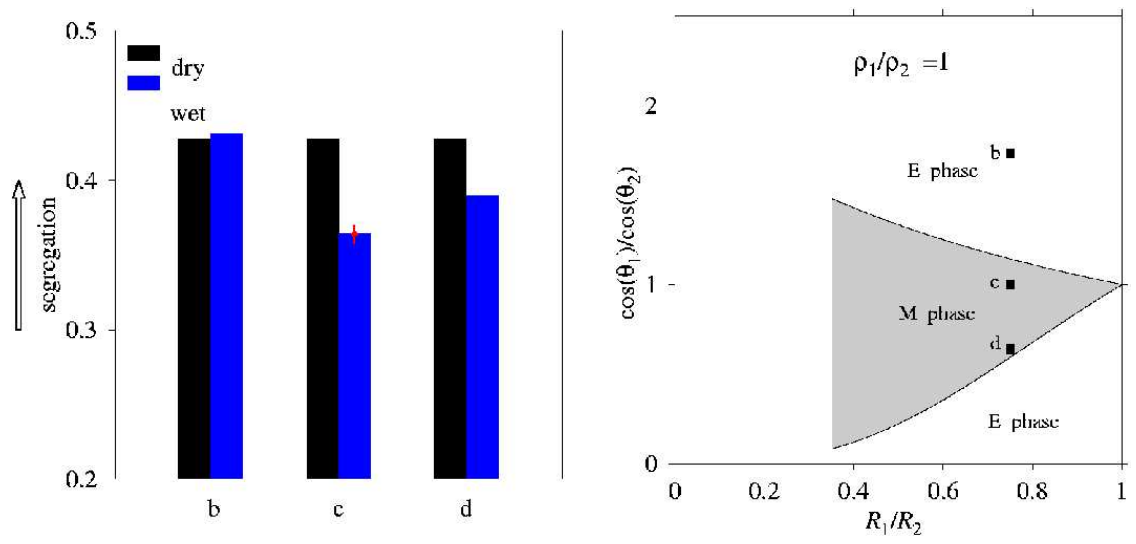

Figure 8: Quantitative Experimental Results and Corresponding Phase Diagram for Sheared Systems. Three trial experiments with surface-treated glass beads (one experiment was run twice as a reproducibility test) yield results in agreement with the extended theoretical predictions. 

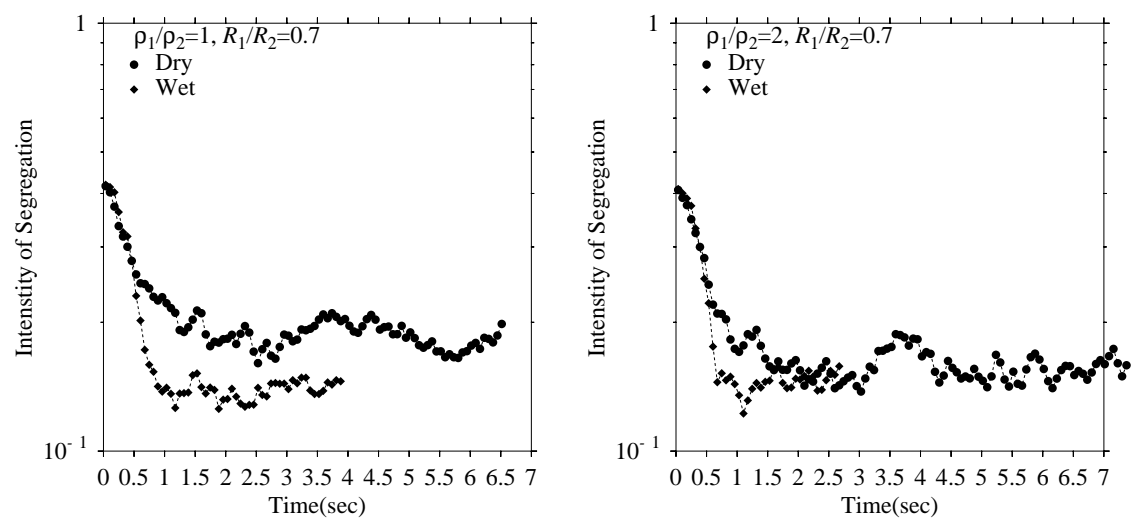

Figure 9: Quantitative Computational Results and Corresponding Phase Diagram for Gas-Solid Systems. Two trial simulations at different density ratios were run and yielded results in reasonable agreement with the extended theoretical predictions (although the mixing in the simulation of the E-phase system was not noticeably hampered by cohesion). Note from Figure 7 that the simulation of $\rho_{1} / \rho_{2}=1$ is in the $M$ phase while $\rho_{1} / \rho_{2}=2$ should be in the E phase. 


\section{Literature References}

[1] V. Reddy. Manuf. Eng., 102:83-86, 1989.

[2] M. Ramlakhan, C. Y. Wu, S. Watano, R. N. Dave, and R. Pfeffer. Powder Technol., 112:137-148, 2000.

[3] P. A. Cundall and O. D. L. Strack. A discrete numerical model for granular assemblies. Geotechnique, 29:47-65, 1979.

[4] J. J. McCarthy, D. V. Khakhar, and J. M. Ottino. Computational studies of granular mixing. Pow. Technol., 109:72-82, 2000.

[5] S.T. Nase, W.L. Vargas, A.A. Abatan, and J.J. McCarthy. Discrete characterization tools for cohesive granular material. Powder Technology, 116:214-223, 2001.

[6] M.J. Rhodes, X.S. Wang, M. Nguyen, P. Stewart, and K. Liffman. Onset of cohesive behavior in gas fluidized beds: a numerical study using dem simulation. Chem. Eng. Sci., 56:4433-4438, 2001.

[7] G. Lian, C. Thornton, and M.J. Adams. Discrete particle simulation of agglomerate impact coalescence. Chem. Eng. Sci., 53:3381-3391, 1998.

[8] T. Mikami, H. Kamiya, and M. Horio. Numerical simulation of cohesive powder in a fluidized bed. Chem. Eng. Sci., 53:1927-1940, 1998.

[9] B.P.B. Hoomans, J.A.M. Kuipers, and W.P.M. van Swaaij. Granular dynamics simulation of segregation phenomena in bubbling gas-fluidized beds. Powder Technology, 109:41-48, 2000.

[10] J.A.M. Kuipers, K.J. van Duin, F.P.H. van Beckum, and W.P.M. van Swaaij. A numerical model for gas-fluidized beds. Chem. Eng. Sci., 47:1913, 1992.

[11] H. Hertz. Ueber die berhrungfester elastischer korper. J. renie ange. Math., 92:1-15, 1881.

[12] R.D. Mindlin. Compliance of elastic bodies in contact. J. Appl. Mech., 16:256-270, 1949.

[13] C. Thornton. Coefficient of restitution for collinear collisions of elasti-perfectly plastic spheres. J. Appl. Mech., 64:383-386, 1997.

[14] P.A. Cundall and O.D.L. Strack. A discrete numerical model for granular assemblies. Géotechnique, 29:47-65, 1979.

[15] R.A. Fisher. On the capillary forces in an ideal soil. J. Agric. Sci., 16:491-505, 1926. 
[16] B.J. Ennis, J. Li, G.I. Tardos, and R. Pfeffer. The influence of viscosity on the strength of an axially strained pendular liquid bridge. Chem. Eng. Sci., 45:3071-3088, 1990.

[17] M.J. Adams and V. Perchard. The cohesive forces between particles with interstitial fluid. Inst. Chem. Eng. Symp., 91:147-160, 1985.

[18] A.J. Goldman, R.G. Cox, and H. Brenner. Slow viscous motion of a sphere parallel to a plan wall i. motion through a quiescent fluid. Chem. Eng. Sci., 22:637-651, 1967.

[19] L.S. Fan and C. Zhu. Principles of Gas Solid Flows. Cambridge University Press, New York, 1998.

[20] R. Di Felice. The voidage function for fluid-particle interaction systems. Int. J. Multiphas. Flow, 20:153-159, 1994.

[21] R.A. Bagnold. Experiments on a gravity-free dispersion of large solid spheres in a newtonian fluid under shear. Proc. R. Soc., 225:4-63, 1954.

[22] H. Li and J.J McCarthy. Controlling cohesive particle mixing and segregation. Phys. Rev. Lett., 90:184301, 2003.

[23] K.D. Kafui, C. Thornton, and M.J. Adams. Discrete particle-continuum fluid modelling for gas-solid fluidised beds. Chem. Eng. Sci., 57:2395-2410, 2002. 